\title{
Electrochemical and Thermal Etching of Indium Tin Oxide by Solid-State Hybrid Organic-Inorganic Perovskites
}

\author{
Ross A. Kerner ${ }^{\mathrm{a}}$ and Barry P. Rand $\mathrm{a}^{\mathrm{a}, \mathrm{b}, *}$ \\ ${ }^{a}$ Department of Electrical Engineering, Princeton University, Princeton, New Jersey 08544, United States \\ ${ }^{b}$ Andlinger Center for Energy and the Environment, Princeton University, Princeton, New Jersey 08544, \\ United States \\ *Corresponding Author Email: brand@princeton.edu
}

\section{Supporting Information}

Materials

The precursors used were methylammonium iodide (Dyesol), formamidinium iodide (Dyesol), $\mathrm{PbI}_{2}$ (98\%, TCI), $\mathrm{InI}_{3}$ (99.998\%, Sigma Aldrich), N,N-dimethylformamide (anhydrous, 99\%, Sigma Aldrich), and toluene (anhydrous, Sigma Aldrich). Indium tin oxide substrates (3 cm x $3 \mathrm{~cm}$ ) were purchased from Lumtec (unpatterned ITO) and Colorado Concept Coatings (patterned for devices).

Experimental

Sample preparation of and thermal annealing was performed inside of a $\mathrm{N}_{2}$ filled glovebox to exclude the effects of moisture and oxygen on perovskite films. The ITO and FTO substrates were cleaned by sequential sonication in DI water + detergent, DI water, acetone, and isopropanol followed by UV-ozone treatment for $15 \mathrm{~min}$ or $\mathrm{O}_{2}$-plasma for $10 \mathrm{~min}$. The MAI:PbI 2 solutions were prepared with $345 \mathrm{mg}$ of $\mathrm{PbI}_{2}$ and $119 \mathrm{mg}$ of MAI per $1 \mathrm{~mL}$ of DMF (129 $\mathrm{mg}$ in the case of FAI). Thin films of $\mathrm{MAPbI}_{3}$ (and $\mathrm{FAPbI}_{3}$ ) were spin coated onto the substrates at $5300 \mathrm{rpm}$ (5300 $\mathrm{rpm} / \mathrm{s}$ acceleration) for $35 \mathrm{~s}$ in an $\mathrm{N}_{2}$ glovebox. A solvent exchange with toluene was performed at 4-6 s after the start of spinning, and the films were annealed at $70{ }^{\circ} \mathrm{C}$ for $20 \min \left(150{ }^{\circ} \mathrm{C}\right.$ for $10-15$ min for $\left.\mathrm{FAPbI}_{3}\right)$. The $\mathrm{PbI}_{2}$ films were spin coated at $4000 \mathrm{rpm}$ (4000 rpm/s acceleration) from a $345 \mathrm{mg} / \mathrm{mL}$ solution in $\mathrm{DMF}$ and annealed at $70{ }^{\circ} \mathrm{C}$ for $20 \mathrm{~min}$. Subsequent thermal annealing/aging of films was performed at temperature/times given in the text.

A Thermo-Scientific K-Alpha X-ray Photoelectron Spectrometer at a base pressure of $5 \times 10^{-8}$ mbar using an Al target (power $=72$ watts), hemispherical analyzer, pass energy of $50 \mathrm{eV}$, and $400 \mu \mathrm{m}$ diameter X-ray beam spot size was used to obtain XPS spectra. The films were transferred to the XPS under vacuum in order to exclude reactions with atmospheric water/oxygen. To resolve the small amount of volatile In in electrically stressed devices, 2 scans (100 ms dwell per point) were rapidly taken at 8 different points and the intensities were averaged. A Veeco diInnova/Bruker Innova Atomic Force Microscope was used to obtain tapping mode AFM images. Cyclic voltammetry measurements were performed with a Keithley $2602 \mathrm{~b}$ inside of an $\mathrm{N}_{2}$ filled glovebox. A step voltage approximation to a linear voltage ramp was used with zero delay time between voltage step and current measurement. 


\section{a) ITO control}

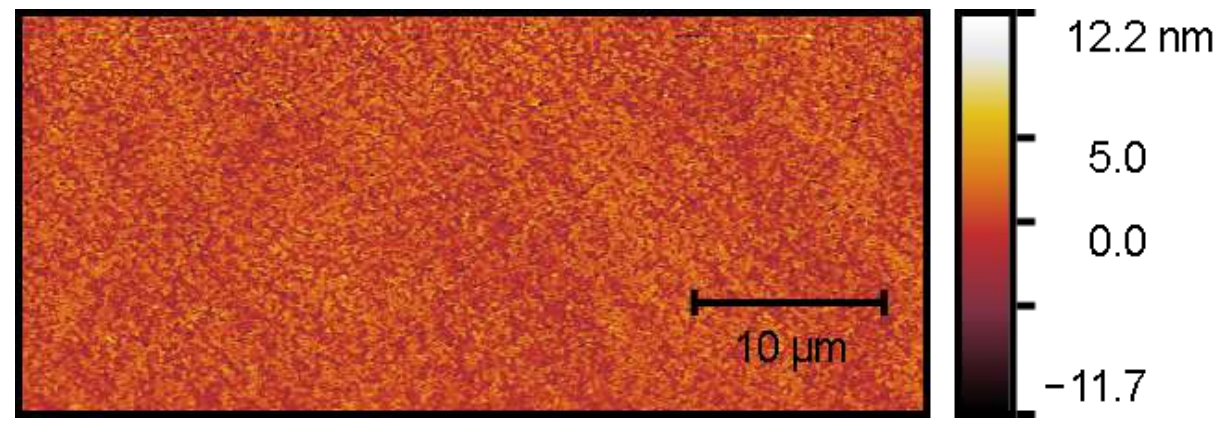

\section{b) ITO - MAl etched}
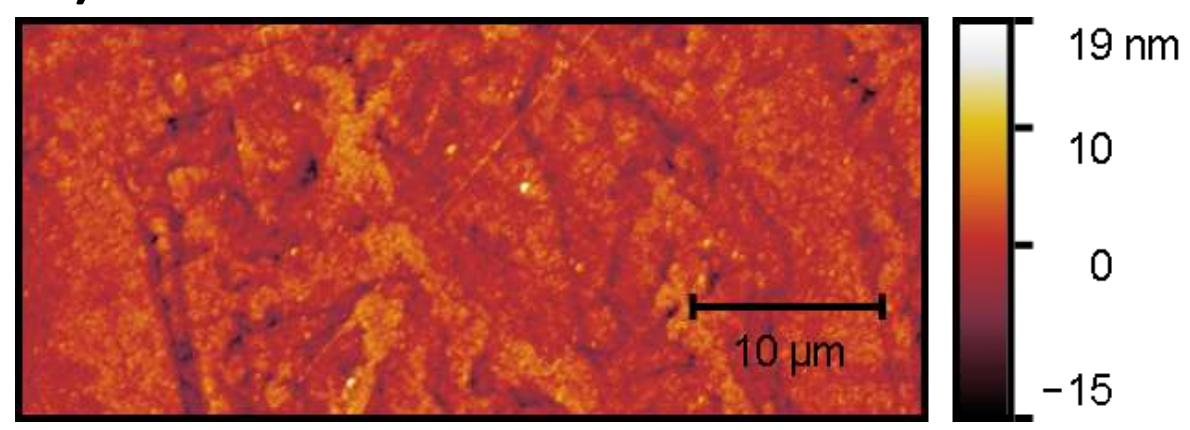

Figure S1. Atomic force micrographs of (a) bare as-received ITO and (b) ITO after etching by solid-state MAI. Etching was performed by drop casting MAI from isopropanol and heating the sample in atmosphere at $95{ }^{\circ} \mathrm{C}$ for $24 \mathrm{~h}$. The excess MAI was washed off with deionized water and isopropanol before performing AFM. 

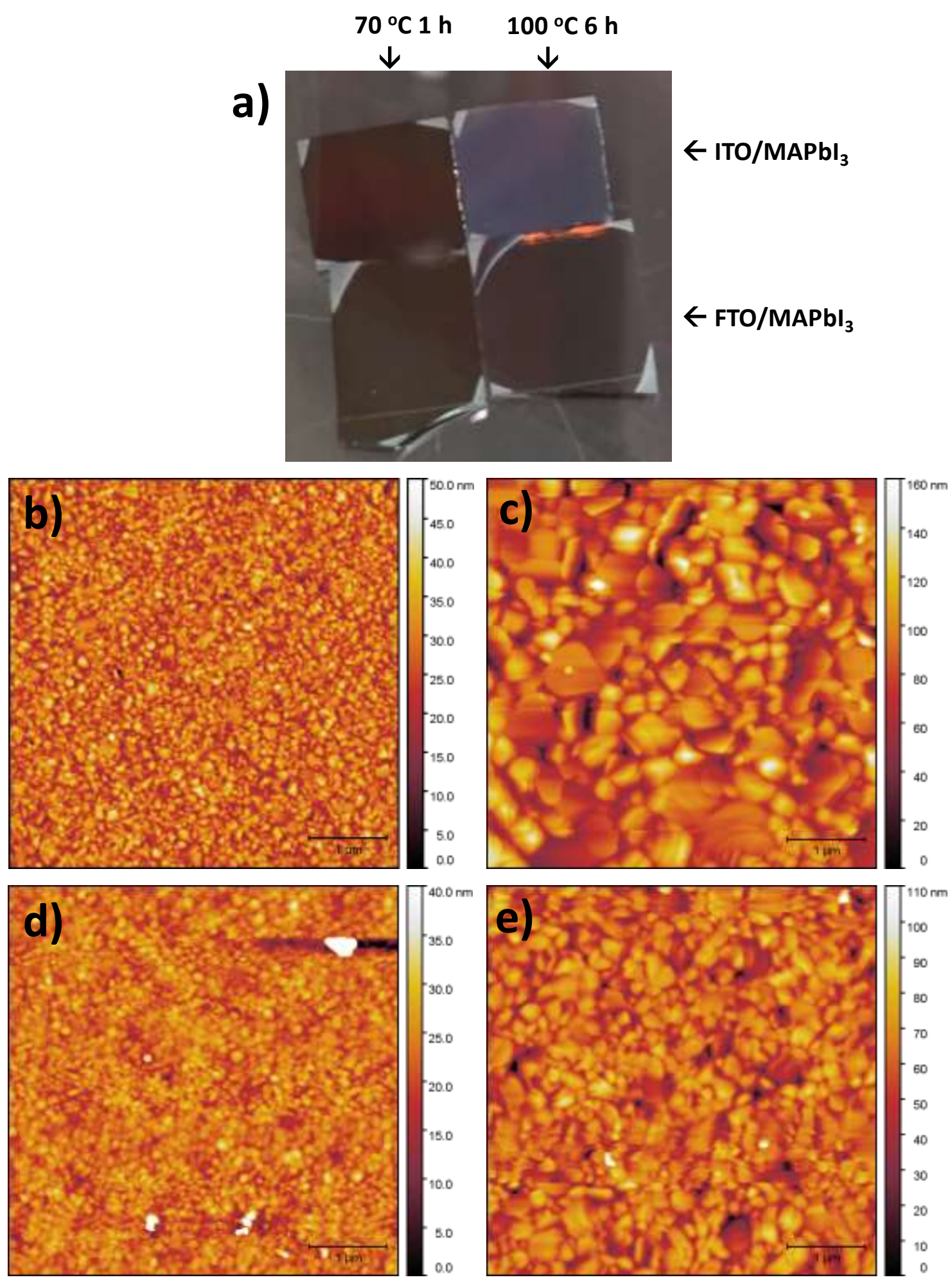

Figure S2. (a) Photograph of $\mathrm{MAPbI}_{3}$ films showing substrate dependent (ITO vs FTO) morphology changes upon annealing. Atomic force micrographs of (b) ITO/MAPbI 3 annealed 70 ${ }^{\circ} \mathrm{C}$ for $20 \mathrm{~min}$, (c) ITO/MAPbI 3 annealed $100{ }^{\circ} \mathrm{C}$ for $6 \mathrm{~h}$, (d) FTO/MAPbI 3 annealed $70{ }^{\circ} \mathrm{C}$ for 20 min, and (e) FTO/MAPbI3 annealed $100{ }^{\circ} \mathrm{C}$ for $6 \mathrm{~h}$. 


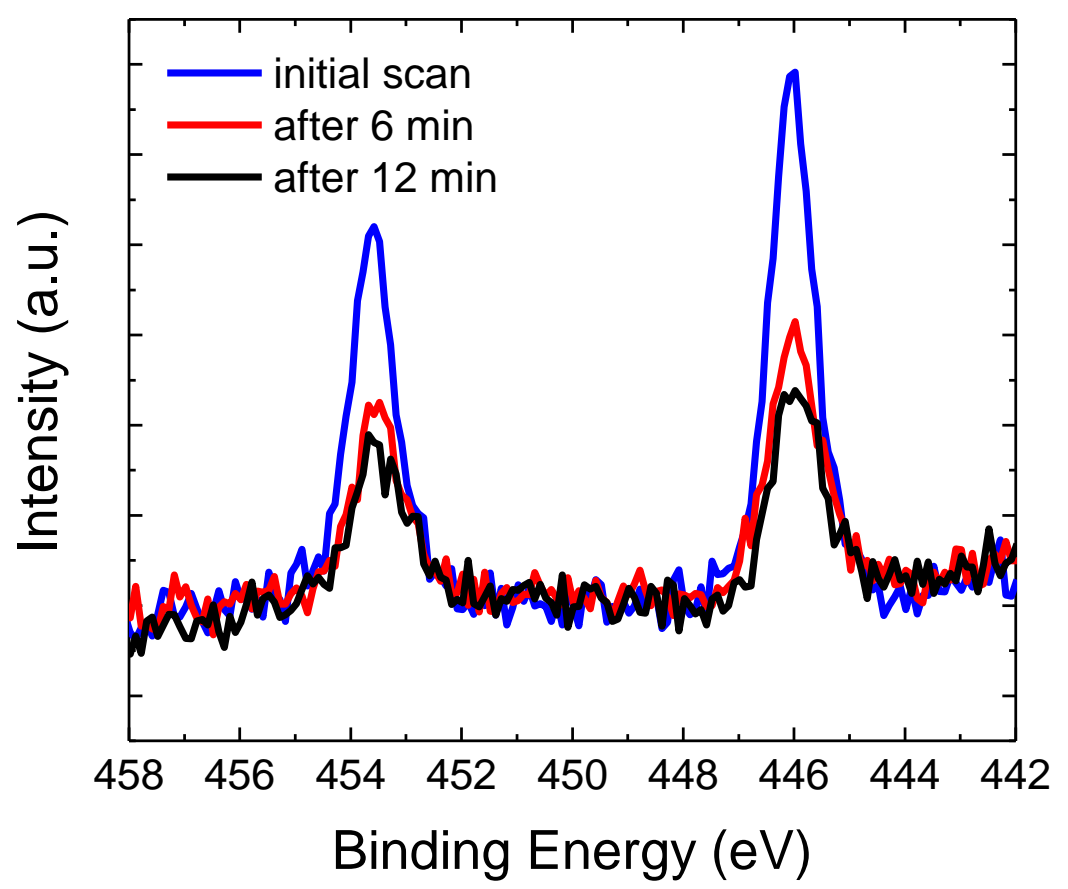

Figure S3. XPS of In 3d region of an FTO/MAPbI $\mathrm{InI}_{3}$ film (MAPbI $3: \mathrm{InI}_{3}=10: 1$ in solution) as a function of measurement time under continuous $\mathrm{X}$-ray irradiation.

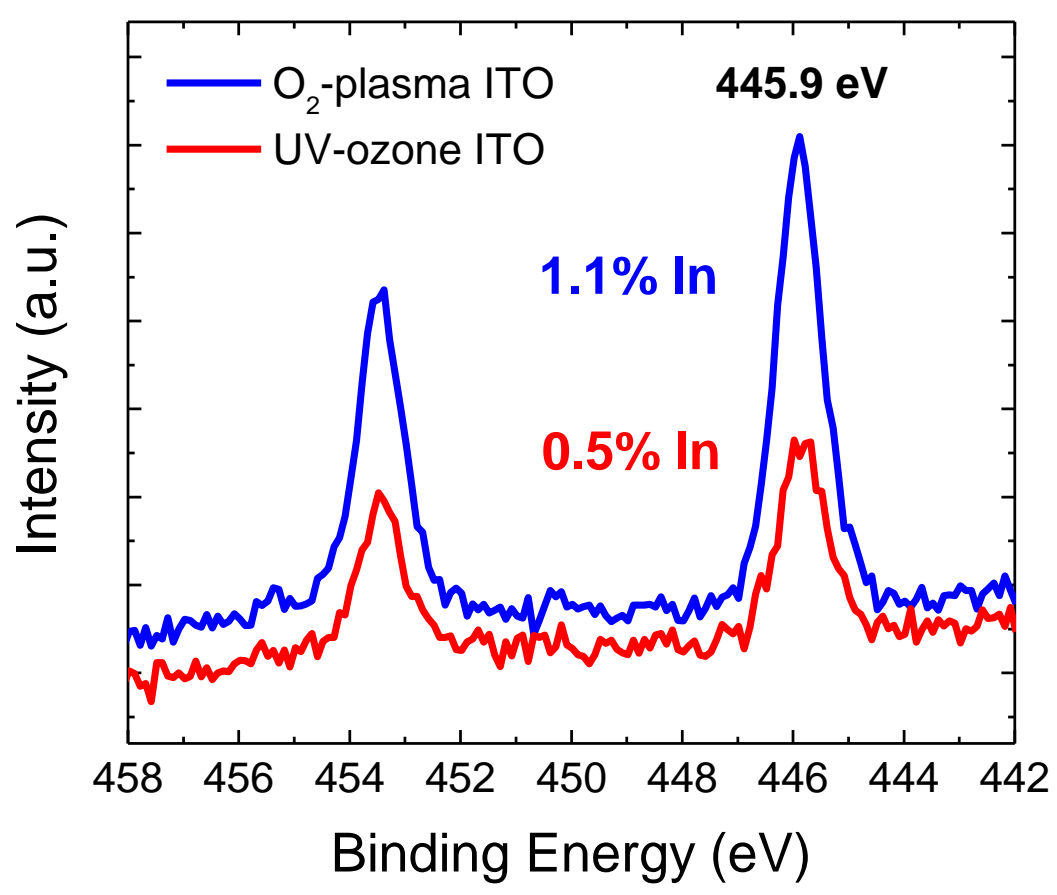

Figure S4. XPS of In 3d region of an ITO/FAPbI 3 samples with different ITO cleaning treatments after annealing $150{ }^{\circ} \mathrm{C}$ for $12 \mathrm{~min}$. 


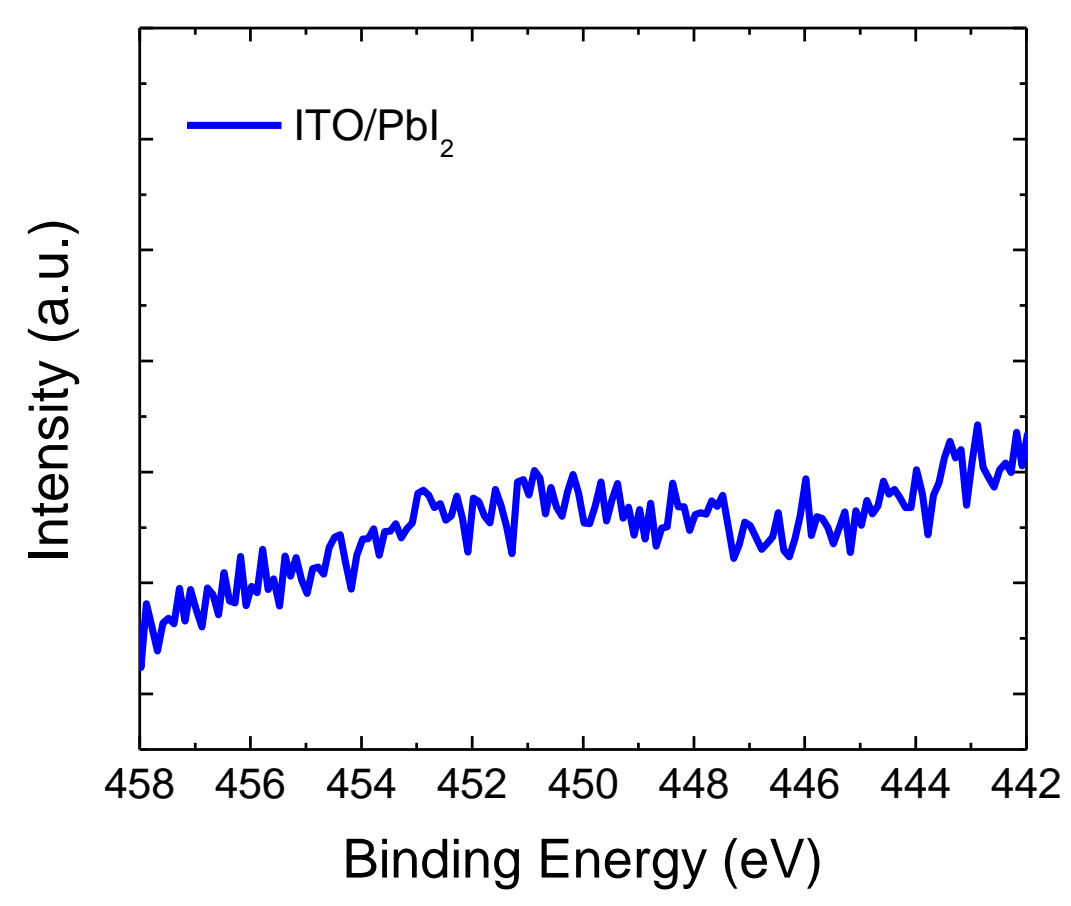

Figure S5. XPS of In 3d region of an ITO $\left(\mathrm{O}_{2}\right.$-plasma $) / \mathrm{PbI}_{2}$ sample annealed $150{ }^{\circ} \mathrm{C}$ for $60 \mathrm{~min}$.

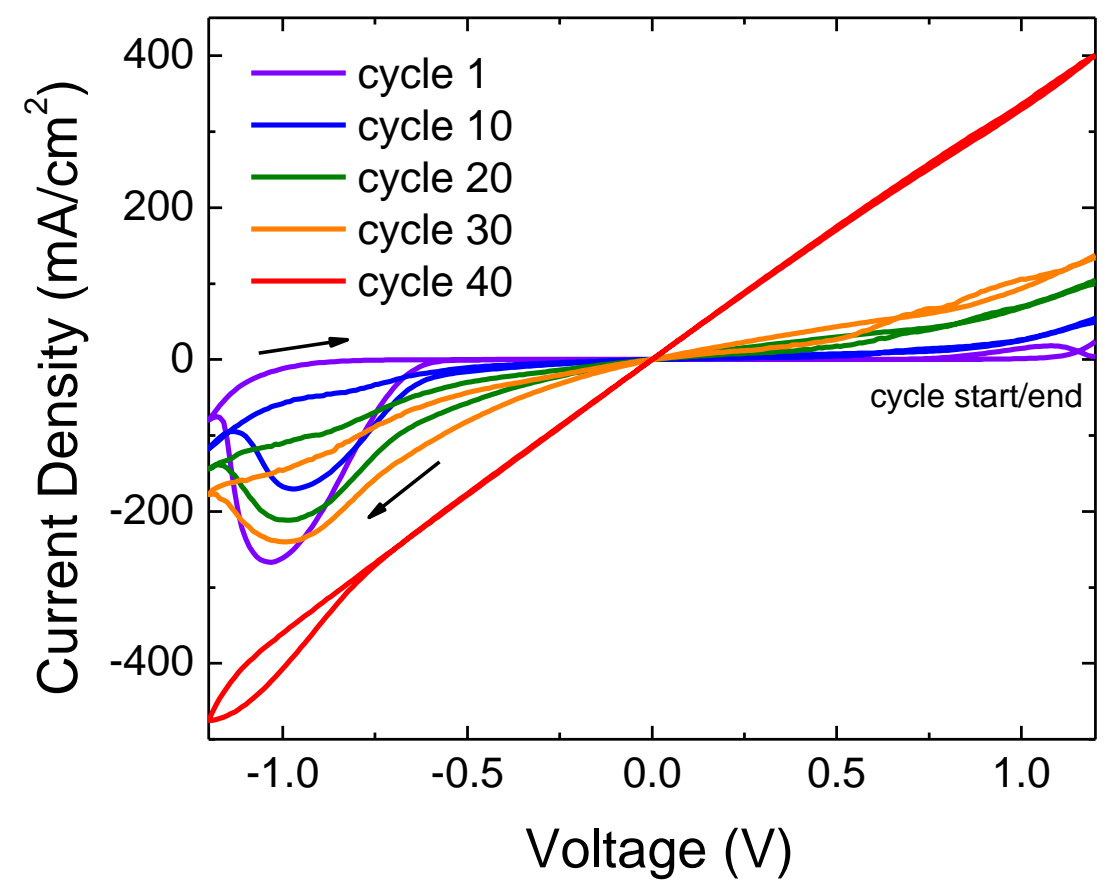

Figure S6. Current density-voltage $(J-V)$ measurements (40 cycles between $-1.2 \mathrm{~V}$ and $+1.2 \mathrm{~V}$ at $10 \mathrm{mV} / \mathrm{s}$ ) of the full range biased $\mathrm{O}_{2}$-plasma ITO/MAPbI $3 / \mathrm{Au}$ device corresponding to Fig. 2 of the main text. The Au electrode is ground. The device was biased until failure (shunting) in the last few cycles. 


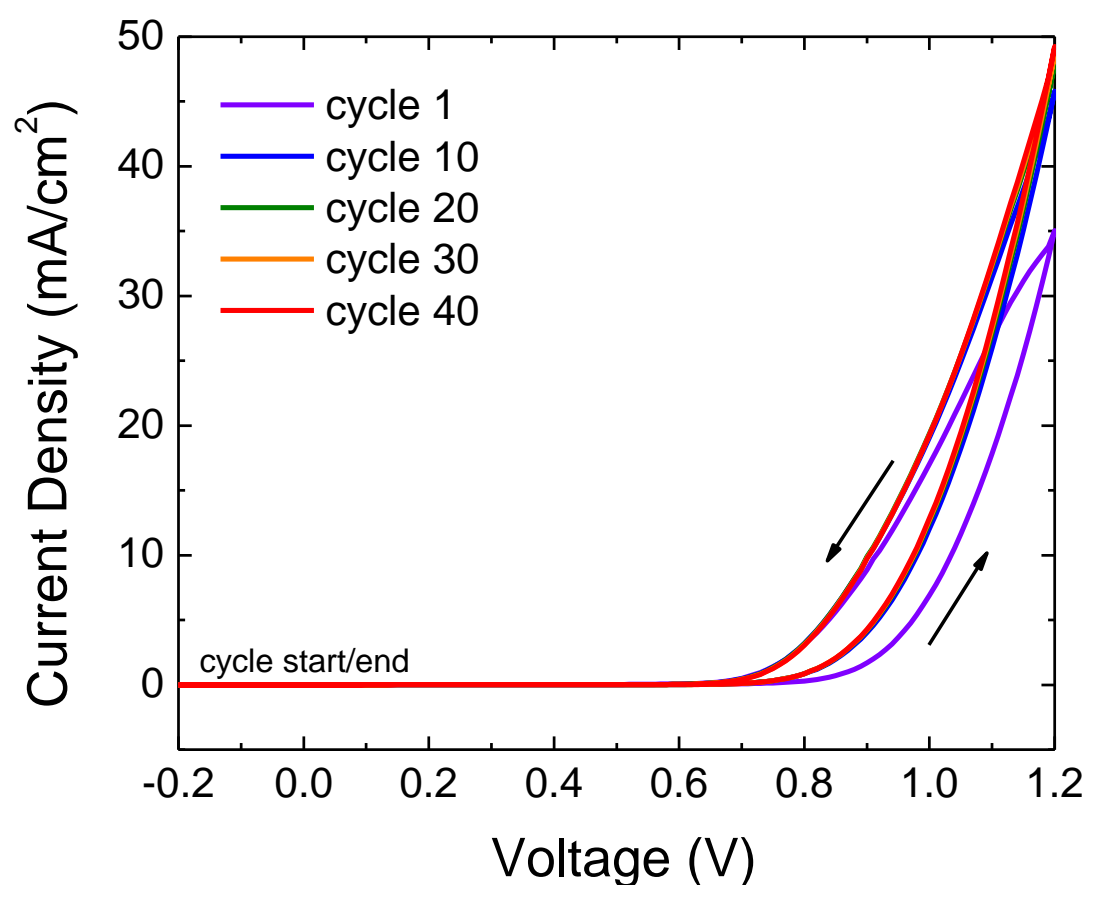

Figure S7. Current density-voltage $(J-V)$ measurements (40 cycles between $+0.2 \mathrm{~V}$ and $-1.2 \mathrm{~V}$ at $10 \mathrm{mV} / \mathrm{s}$ ) of the anodic biased $\mathrm{O}_{2}$-plasma ITO/MAPbI $3 / \mathrm{Au}$ device corresponding to Fig. 2 of the main text. The Au electrode is ground.

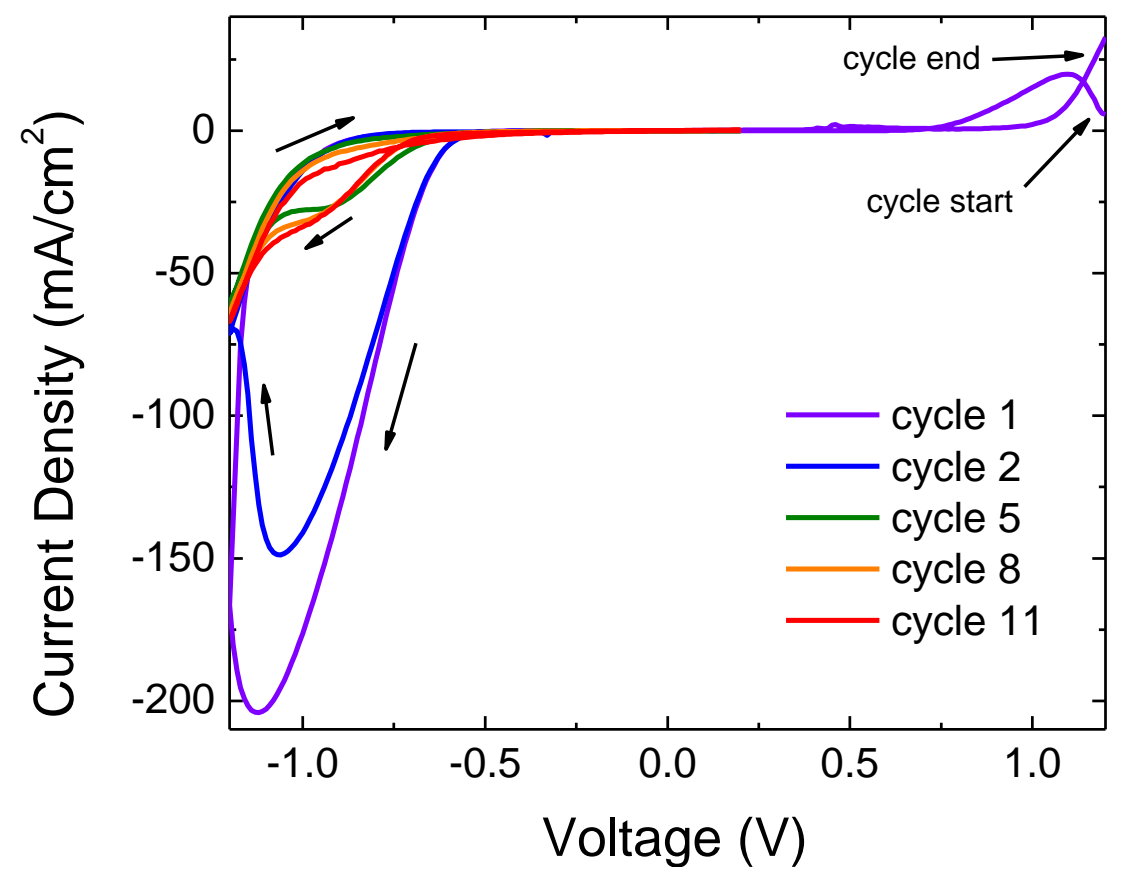

Figure S8. Current density-voltage $(J-V)$ measurements (initial cycle $+1.2 \mathrm{~V}$ to $-1.2 \mathrm{~V}$ followed by 10 cycles between $+0.2 \mathrm{~V}$ and $-1.2 \mathrm{~V}$ all at $10 \mathrm{mV} / \mathrm{s}$ ) of the cathodic biased $\mathrm{O}_{2}$-plasma ITO/MAPbI $3 / \mathrm{Au}$ device corresponding to Fig. 2 of the main text. The Au electrode is ground. Note that indium leaching was cathodically induced despite cycling the device only 11 times compared to 40 cycles for the anodically biased device. 

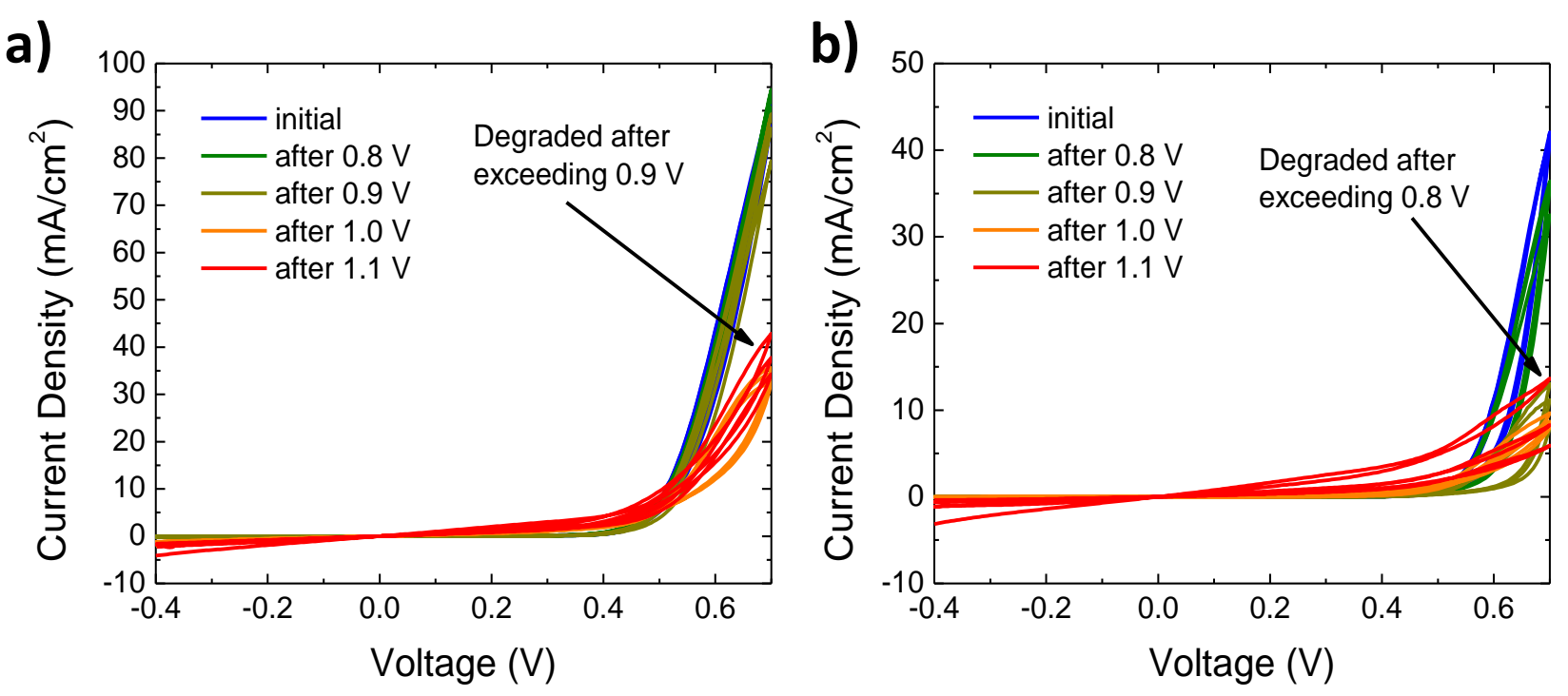

Figure S9. Cyclic $J-V$ characteristics of (a) UV-ozone versus (b) $\mathrm{O}_{2}$-plasma treated ITO/MAPbI $3 / \mathrm{Au}$ devices showing enhanced stability of the UV-ozone treated ITO extending the electrochemical stability window to $0.9 \mathrm{~V}$ compared to $0.7-0.8 \mathrm{~V}$ for $\mathrm{O}_{2}$-plasma ITO (ITO is ground in these plots). The measurement protocol was 3 cycles at $1 \mathrm{mV} / \mathrm{s}$ with scan ranges that increased incrementally in the following order:

- $-0.4 \mathrm{~V}$ to $0.7 \mathrm{~V}$ r

- $-0.4 \mathrm{~V}$ to $0.8 \mathrm{~V}$

- $-0.4 \mathrm{~V}$ to $0.7 \mathrm{~V}$

- $-0.4 \mathrm{~V}$ to $0.9 \mathrm{~V}$

- $-0.4 \mathrm{~V}$ to $0.7 \mathrm{~V}$

Shown in Fig. S9

- $-0.4 \mathrm{~V}$ to $1.0 \mathrm{~V}$

- $-0.4 \mathrm{~V}$ to $0.7 \mathrm{~V}$

- $-0.4 \mathrm{~V}$ to $1.1 \mathrm{~V}$

- $-0.4 \mathrm{~V}$ to $0.7 \mathrm{~V}$

The electrochemical stability window of this device has been determined to be approximately -0.5 $\mathrm{V}$ to $0.9 \mathrm{~V}$. Shown in Fig. S9 are all the scans from -0.4 to $0.7 \mathrm{~V}$ to compare only $J$ - $V$ characteristics within the stability window. 


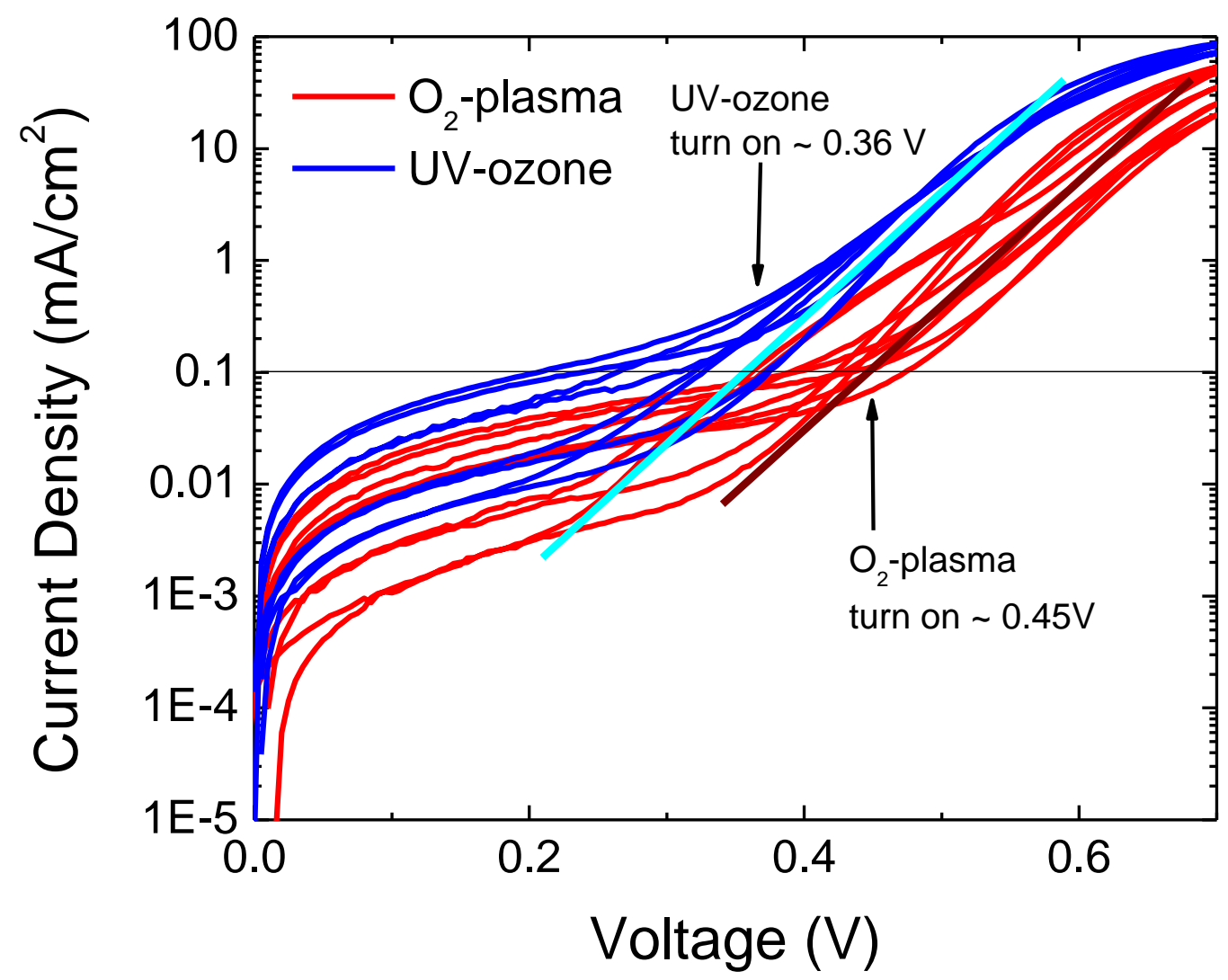

Figure S10. Forward bias diode $J-V$ characteristics of UV-ozone vs $\mathrm{O}_{2}$-plasma treated ITO/MAPbI $3 / \mathrm{Au}$ devices. Shown are 2 representative pixels from 2 separate substrates for each type of device (8 pixels total). Note that within the electrochemical stability window of this device, forward bias is the Au positively biased with respect to ITO (i.e. ITO is ground in this Figure in contrast to Figures S6-S8). The given turn-on voltages were defined from the forward scans at a current density of $0.1 \mathrm{~mA} / \mathrm{cm}^{2}$. The drawn lines are a guide to the eye for a representative turn-on voltage. 